\title{
Rucaparib and its Usage Areas
}

\author{
Melda Ateş', Nejan Saygın²
}

\section{DNA REPAIR AND PARP INHIBITION}

Most anticancer drugs cause cell death by damaging deoxyribonucleic acid (DNA). DNA repair mechanisms correct this damage and create resistance to drugs. It has been observed that these repair mechanisms are expressed much more in cancer cells. Inhibiting the enzymes that repair DNA, inhibits the repair of the cancer cell and stops its proliferation, causes cancer cell apoptosis (programmed cell death) (Figure 1). ${ }^{[1-4]}$

Poly ADP-ribose polymerases (PARP) are a family of proteins involved in cellular processes such as DNA repair, apoptosis, and genomic stability. The first PARP enzyme was discovered in the 1960s and 18 PARP enzymes have been identified to date. PARP-1, one of the most important members of this family of enzymes, is localized in the nucleus. This enzyme with 1014 amino acids encoded in the first chromosome; it consists of three main domains: DNA binding domain, the central auto-modification domain, and the catalytic domain (Figure 2). ${ }^{[5-10]}$

PARP-1 repairs single-strand breaks (SSB) by base excision repair (BER). The enzyme is activated by $\mathrm{NAD+}$ (nicotinamide adenine dinucleotide) when it is attached to a single strand breaks by zinc-fingered

'Demiroğlu Bilim University Medical School, Istanbul, Turkey
${ }^{2}$ Çukurova University Pharmacy School, Adana, Turkey

Correspondence: Melda Ateş. Demiroğlu Bilim Üniversitesi Tıp Fakültesi, 34394 Şişli, İstanbul, Türkiye.

E-mail: meldaates@outlook.com

Cite this article as: Ateş M, Saygın N. Rucaparib and its Usage Areas. JEB Med Sci 2020;1(2):47-51.

doi: $10.5606 /$ jebms.2020.75611

Received : October 10, 2020

Published online : November 09, 2020

@2020 Journal of Experimental and Basic Medical Sciences. All rights reserved.

\begin{abstract}
Anticancer drugs aim to get rid of cancer cells by causing DNA damage but DNA repair mechanisms correct this damage and save the cancer cell. PARP is an enzyme involved in DNA repair. Rucaparib inhibits this enzyme, disrupts cell repair and drags the cancer cell to apoptosis. Studies have shown that tumors with mutated or epigenetically silenced BRCA1/2 genes are sensitive to Rucaparib. Rucaparib 's anticancer activity has been observed in various tumor types, including ovarian, breast and pancreatic cancers. Rucaparib has been approved by the FDA for the treatment of patients with the recurrent epithelial ovary, fallopian tube, primary peritoneal cancer and advanced ovarian cancer associated with BRCA1/2 mutations (germline and/or somatic).

Keywords: Amino acid. DNA repair, PARP inhibitors.
\end{abstract}

domain. This poly (ADP-ribosyl)ation provides the negativity necessary for the formation of the multiprotein complex of base excision repair (BER) in SSB. (Figure 3). ${ }^{[1,5]}$

PARP inhibitors are most commonly used in the treatment of breast and ovarian cancers. Due to the failure to repair SSB which occurs spontaneously with PARP-1 inhibition, double-strand breaks (DSB) begin to form. This DSB which normally repaired by homologous recombination (HR) repair, remain irreversibly damaged in cancer cells mutated in at least one of the BRCA1 or BRCA2 (tumor-suppressor gene) genes. ${ }^{[11]}$

PARP inhibitors can be administered in two ways in the clinic. One of them is combined therapy with chemotherapy and the other is as a single-agent treatment in patients with homologous recombination repair (HRR) damage (eg BRCA mutation). ${ }^{[8]}$

\section{RUCAPARIB}

Rucaparib is a small molecule potent PARP inhibitor taken orally. ${ }^{[12]}$ Rucaparib camicylate (CO338; 


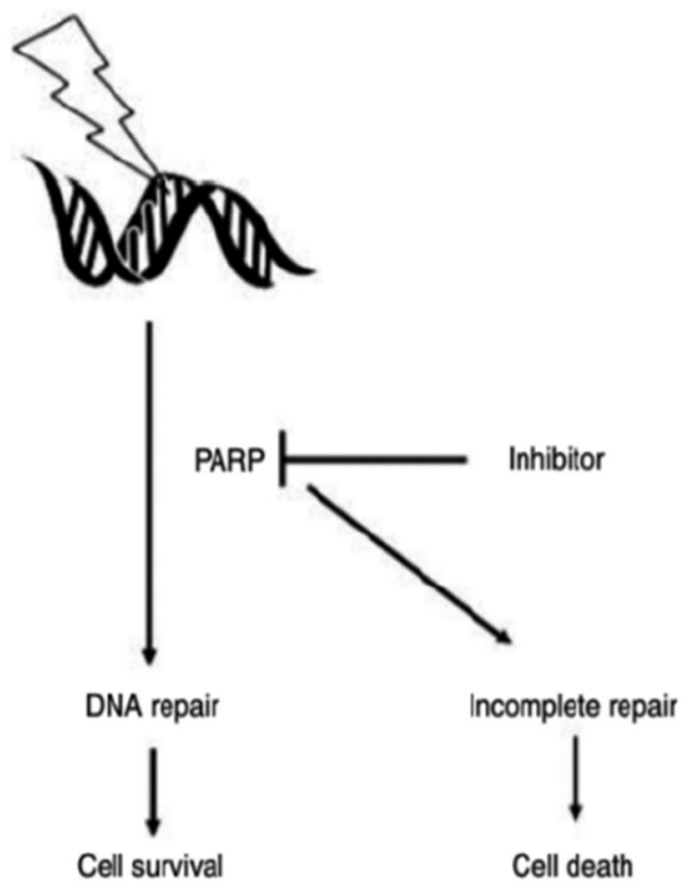

Figure 1. Single chain fractures that cannot be repaired due to PARP enzyme inhibition cause cell death. ${ }^{[4]}$

PARP: Poly ADP-ribose polymerases.

8-fluoro-2- $\{4$ - [(methylamino) methyl $]$ phenyl $\}$ -1,3,4,5-tetrahydro-6H-azepino $[5,4,3-\mathrm{cd}]$ indole6-one ((1S, 4R) -7,7-dimethyl-2-oxobicyclo [2.2.1] hept1-yl) methanesulfonic acid salt) was formulated into oral tablets. ${ }^{[13]}$

Oral cancer therapies often exhibit very different pharmacokinetics due to varying absorption levels, leading to unpredictable toxicity and efficacy. Rubraca, a PARP inhibitor (PARPi), was developed to

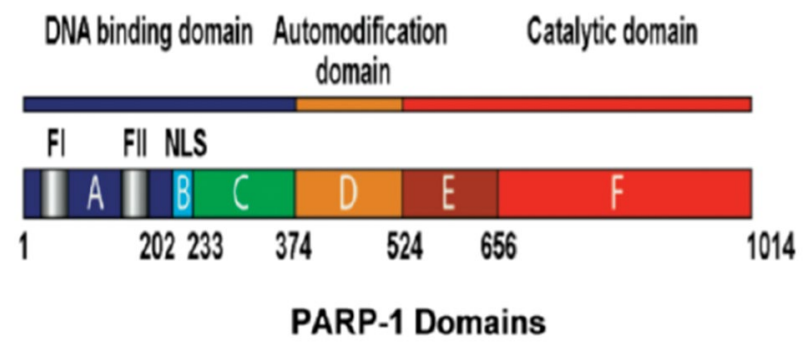

Figure 2. The zinc-finger DNA binding domains combine DNA fractures and the central domain allows modification. The catalytic domain of the PARP-1 enzyme (carrying the carboxylic acid group) is the target region while most drugs are developed. ${ }^{[8,10]}$

DNA: Deoxyribonucleic acid; PARP: Poly ADP-ribose polymerases. act on tumors that have homologous recombination repair defects with a predetermined target plasma level threshold. ${ }^{[12]}$

Rucaparib can be use as fasting or full, but it has different pharmacokinetic parameters when taken with food (compared to fasting) due to its solubility in the small intestine. The median half-life is 17 hours and the median time to reach maximum concentration is 1.9 hours, which can be extended to 2.5 hours after a high-fat meal. However, it is thought that food interaction doesn't have a clinically significant effect on pharmacokinetics.Tmax exhibits good oral absorption ranging from 1 to 6 hours. ${ }^{[14]}$

The cytochrome P450 enzymatic pathway is responsible for Rucaparib's metabolism (primarily metabolized by CYP2D6). Slow metabolic conversion rates were observed in incubation with human hepatocytes and liver microsomes when the metabolism of Rucaparib was examined in vitro. ${ }^{[12]}$ The Phase I-II study "study 10" was conducted in severely pretreated patients with Oral Rucaparib with advanced solid tumors. The dose toxicity

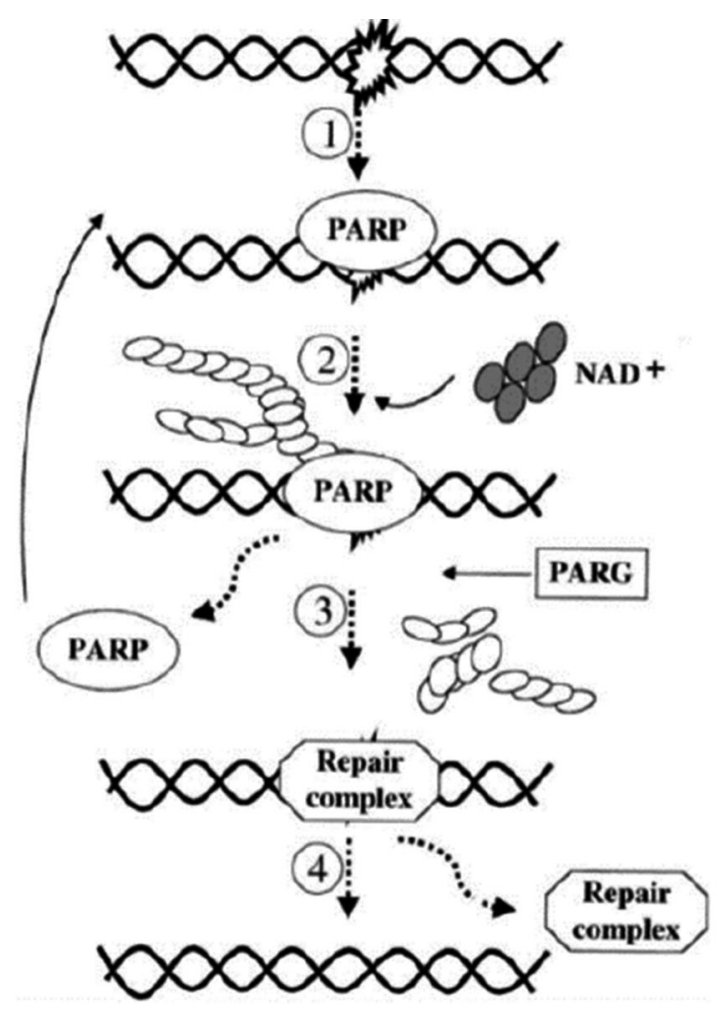

Figure 3. The PARP enzyme activates with NAD+ and repairs single strain breaks in DNA. ${ }^{[5]}$

PARP: Poly ADP-ribose polymerases; NAD: Nicotinamide adenine dinucleotide; DNA: Deoxyribonucleic acid. 
and pharmacokinetic assessments documented in the Phase- 1 study concluded that $600 \mathrm{mg}$ twice daily Rucaparib is safe and manageable and is the recommended dose for future studies. Rucaparib $600 \mathrm{mg}$ twice daily showed strong antitumor activity in patients with platinum-sensitive ovarian cancer and germline BRCA $1 / 2$ mutation. These results support the further clinical and translational investigation of Rucaparib in tumors with HR (homologous recombination) repair defect, potentially expanding its applicability beyond cancers with BRCA mutations. ${ }^{[14]}$

\section{RUCAPARIB USAGE AREAS}

Rubraca's anticancer activity has been observed in various tumor types, including ovarian, breast and pancreatic cancers. ${ }^{[14-16]}$

Rucaparib has been approved by FDA in the maintenance treatment of patients with recurrent epithelial ovarian cancer, fallopian tube cancer or primary peritoneal cancer, who have a complete or partial response to platinum-based chemotherapy and approved as monotherapy in the treatment of patients with advanced ovarian cancer associated BRCA1 or BRCA2 mutations (germline and/or somatic) with at least two chemotherapy-treated. ${ }^{[12]}$

Approximately $10-15 \%$ of breast and ovarian cancers occur due to BRCA1 and BRCA2 mutations. In preclinical studies, tumors with mutated or epigenetically silenced BRCA1/2 genes are sensitive to Rucaparib. ${ }^{[17]}$

In 3 clinical studies conducted between 2013 and 2016, Rucaparib has been shown to have singleagent anticancer activity in patients with high-grade ovarian carcinoma. These; Study 10 (a phase I/II treatment trial), ARIEL 2 (a phase II treatment trial), and ARIEL 3 (a phase III switch maintenance trial) are clinical trials. ${ }^{[12,15,18]}$

Oral Rucaparib was clinically evaluated for the first time in the Phase I-II studies, Study 10, and the Phase I study determined the maximum tolerable dose (600 mg twice daily) and the pharmacokinetics of the drug. In the Phase II study, Rucaparib twice daily was administered to 42 patients with platinumsensitive, high-grade ovarian cancer due to the germline BRCA $1 / 2$ mutation. The overall response rate evaluated by the researcher was $59.5 \%$ according to the RECIST criteria ${ }^{[19]}$ and $83.3 \%$ according to the RECIST/GCIG CA-125 $5^{[20]}$ criteria. The median response time was 7.8 months. (95\% Cl (confidence interval): 5.6-10.5). ${ }^{[12]}$

In the light of these studies, the ARIEL2, phase II trial was initiated to evaluate the safety and efficacy of Rucaparib in platinum-sensitive, recurrent highgrade ovarian cancer patients who had received one or more chemotherapy regimens or who had previously received 3 or 4 chemotherapy regimens (prior chemotherapy regimens). 204 patients were treated with $600 \mathrm{mg}$ oral Rucaparib twice daily for 28-day cycles during the disease (or until the drug was discontinued for a different reason). Genomic loss of heterozygosity (LOH) was chosen as the defining biomarker for homologous recombination deficiency (HRD). The predetermined limitation for the high definition of $\mathrm{LOH}$ was defined as $\mathrm{LOH} 14 \%$. Patients were classified into three groups according to their HRDs: BRCA mutations (germline and somatic), BRCA wild-type and high LOH (high LOH group), and finally BRCA wild-type and low LOH (low LOH group).

\begin{tabular}{|c|c|c|c|c|c|c|}
\hline & \multicolumn{3}{|c|}{$\begin{array}{c}\text { Confirmed objective responses by } \\
\text { RECIST }\end{array}$} & \multicolumn{3}{|c|}{$\begin{array}{c}\text { Objective responses by combined } \\
\text { RECIST and CA-125 }\end{array}$} \\
\hline & $\mathrm{n}$ & $\%$ & Min-Max & $\mathrm{n}$ & $\%$ & Min-Max \\
\hline BRCA mutant $(n=40)$ & 32 & 80 & $64-91$ & 34 & 85 & $70-94$ \\
\hline Germline mutation $(n=20)$ & 17 & 85 & $62-97$ & 17 & 85 & $62-97$ \\
\hline Somatic mutation $(n=19)$ & 14 & 74 & $49-91$ & 16 & 84 & $60-97$ \\
\hline Indeterminate $(\mathrm{n}=1)$ & 1 & 100 & $3-100$ & 1 & 100 & $3-100$ \\
\hline BRCA1 mutation $(n=29)$ & 23 & 79 & $60-92$ & 25 & 86 & $68-96$ \\
\hline BRCA2 mutation $(n=11)$ & 9 & 82 & $48-98$ & 9 & 82 & $48-98$ \\
\hline $\mathrm{PFI} \geq 6$ to $<12$ months $(\mathrm{n}=23)$ & 20 & 87 & $66-97$ & 20 & 87 & $66-97$ \\
\hline $\mathrm{PFI} \geq 12$ months $(n=17)$ & 12 & 71 & $44-90$ & 14 & 82 & $57-96$ \\
\hline BRCA wild-type and LOH high $(n=82)$ & 24 & 29 & $20-40$ & 36 & 44 & $33-55$ \\
\hline BRCA wild-type and LOH low ( $n=70)$ & 7 & 10 & $4-20$ & 14 & 20 & $11-31$ \\
\hline BRCA wild-type and LOH not classified $(n=12)$ & 4 & 33 & $10-65$ & 7 & 58 & $28-85$ \\
\hline
\end{tabular}

LOH: Loss of heterozygosity. 
192 of the treated patients were classified into one of the three HRD subgroups; The BRCA mutant $(n=40)$, high LOH $(n=82)$, low LOH $(n=70)$, or unclassified $(n=12) .{ }^{[15]}$

After Rucaparib treatment, median progressionfree survival (PFS) was 12.8 months $(95 \% \mathrm{Cl}$ : 9.014.7) in the BRCA mutated subgroup, 5.7 months (5.3-7.6) in the LOH high subgroup, and 5.2 months in the low subgroup (3.6-5.5). PFS was longer in the BRCA mutant subgroup (HR: $0.27 ; 95 \% \mathrm{Cl}: 0.16-$ $0.44 ; \mathrm{p}<0.0001)$ and in the LOH high subgroup (HR: $0.62 ; 0.42-0.90 ; p=0.011$ ), longer than the LOH low subgroup. (Table 1) ${ }^{[15]}$

The proportion of patients receiving the RECIST response was significantly higher in the $B R C A$ mutated $(p<0.0001)$ and LOH high $(p=0.0033)$ subgroups compared to the LOH low subgroup. The proportion of patients who responded to treatment was similar regardless of whether the BRCA mutation was germline or somatic, or whether the patient had a BRCA1 or BRCA2 mutation. According to the approved RECIST and CA-125 combined responses, BRCA mutated $(p<0.0001)$ and LOH high $(p=0.0018)$ subscales showed significantly more frequent response to treatment than the LOH low subgroup. ${ }^{[15]}$

ARIEL 3 is a randomized, double-blind, placebocontrolled phase III trial involving patients with platinum-sensitive high-grade serous or endometrioid ovaries, primary peritoneal or fallopian tube carcinoma. All patients included in the study received at least two platinum-based chemotherapy regimens, and partially or fully responded to their last platinum-based regimen. The study was conducted with 375 randomly selected patients who were treated with $600 \mathrm{mg}$ oral Rucaparib twice a day in 28-day cycles and while 189 patients were in the placebo group (564 patients in total). Patients were classified into three groups: Germline or somatic BRCA mutations (130 Rucaparib, 66 placebo), HRD positive (BRCA wild-type and high LOH carcinomas; 236 Rucaparib, 118 placebo) and the intention to treat population (randomly assigned patients). The predetermined limitation for the high definition of $\mathrm{LOH}$ was defined as $\mathrm{LOH} \geq 16 \%{ }^{\left[{ }^{[18]}\right.}$

Median PFS (months; $95 \% \mathrm{Cl}$ ) in the BRCA mutant group after Rubraca treatment, 16.6 months (13.4-22.9) in the treated group with BRCA mutation, 5.4 months in the placebo group (3.4-6.7, [HR: 0.23 ; 95\% Cl: 0.160.34; $p<0.0001]$ ). In patients with HRD carcinoma, median PFS was 13.6 months (10.9-16.2) in the treated group, 5.4 months in placebo (5.1-5.6; 0.32 [0.24-0.42]; $p<0.0001)$. The intention to treat subgroups median PFS was 10.8 months (8.3-11.4) in the Rucaparib group and 5.4 months in the placebo group $(5.3-5.5 ; 0.36$ [0.30-0.45]; $p<0.0001) .{ }^{[18]}$

In all subgroups, the benefit of Rucaparib was observed in PFS regardless of the volume of the disease, responses to chemotherapy, and the status of LOH or BRCA mutations compared to placebo. In this study, the overall response rate was increased in all populations with measurable disease treated with Rucaparib. ${ }^{[18]}$

\section{Declaration of conflicting interests}

The authors declared no conflicts of interest with respect to the authorship and/or publication of this article.

\section{Funding}

The authors received no financial support for the research and/or authorship of this article.

\section{REFERENCES}

1. Drew Y, Plummer R. PARP inhibitors in cancer therapy: two modes of attack on the cancer cell widening the clinical applications. Drug Resist Updat 2009;12:153-6.

2. Nickoloff JA, Jones $\mathrm{D}$, Lee $\mathrm{SH}$, Williamson EA, Hromas R. Drugging the cancers addicted to DNA repair. J Natl Cancer Inst 2017;109:djx059.

3. Staibano S, Pepe S, Lo Muzio L, Somma P, Mascolo M, Argenziano $\mathrm{G}$, et al. Poly(adenosine diphosphate-ribose) polymerase 1 expression in malignant melanomas from photoexposed areas of the head and neck region. Hum Pathol 2005;36:724-31.

4. Javle M, Curtin NJ. The role of PARP in DNA repair and its therapeutic exploitation. Br J Cancer 2011;105:1114-22.

5. Herceg Z, Wang ZQ. Functions of poly(ADP-ribose) polymerase (PARP) in DNA repair, genomic integrity and cell death. Mutat Res 2001;477:97-110.

6. Zhu Q, Wang X, Chu Z, He G, Dong G, Xu Y. Design, synthesis and biological evaluation of novel imidazo[4,5-c]pyridinecarboxamide derivatives as PARP1 inhibitors. Bioorg Med Chem Lett 2013;23:1993-6.

7. Zhou D, Chu W, Xu J, Jones LA, Peng X, Li S, et al. Synthesis, [18F] radiolabeling, and evaluation of poly (ADP-ribose) polymerase-1 (PARP-1) inhibitors for in vivo imaging of PARP-1 using positron emission tomography. Bioorg Med Chem Lett 2014;22:1700-7.

8. Plummer R. Poly(ADP-ribose)polymerase (PARP) inhibitors: from bench to bedside. Clin Oncol ( $R$ Coll Radiol) 2014;26:250-6.

9. Bürkle, A. Poly (ADP-ribosyl) ation. New York: Springer Science \& Business Media; 2008.

10. TaoZ,GaoP, Liu HW. Identification of the ADP-ribosylation sites in the PARP-1 automodification domain: analysis and implications. J Am Chem Soc 2009;131:14258-60.

11. Giannini G, Battistuzzi G, Vesci L, Milazzo FM, De Paolis F, Barbarino M, et al. Novel PARP-1 inhibitors based on a 
2-propanoyl-3H-quinazolin-4-one scaffold. Bioorg Med Chem Lett 2014;24:462-6.

12. Shapiro Gl, Kristeleit RS, Burris HA, LoRusso P, Patel MR, Drew $Y$, et al. Pharmacokinetic study of rucaparib in patients with advanced solid tumors. Clin Pharmacol Drug Dev 2019;8:107-18.

13. National Center for Biotechnology Information. PubChem Database. Rucaparib (Camsylate), $C I D=121490161$. Available at: https://pubchem.ncbi.nlm. nih.gov/compound/Rucaparib-Camsylate

14. Kristeleit R, Shapiro Gl, Burris HA, Oza AM, LoRusso P, Patel MR, et al. A Phase I-Il Study of the Oral PARP Inhibitor Rucaparib in Patients with Germline BRCA1/2Mutated Ovarian Carcinoma or Other Solid Tumors. Clin Cancer Res 2017;23:4095-106.

15. Swisher EM, Lin KK, Oza AM, Scott CL, Giordano H, Sun $\mathrm{J}$, et al. Rucaparib in relapsed, platinum-sensitive highgrade ovarian carcinoma (ARIEL2 Part 1): an international, multicentre, open-label, phase 2 trial. Lancet Oncol 2017;18:75-87.

16. Domchek $S M$, Hendifar AE, McWilliams RR, Geva R, Epelbaum R, Biankin A, et al. RUCAPANC: An openlabel, phase 2 trial of the PARP inhibitor rucaparib in patients (pts) with pancreatic cancer (PC) and a known deleterious germline or somatic BRCA mutation. J Clin Oncol 2016;34(15 Suppl):4110-4110.

17. Drew $Y$, Mulligan EA, Vong WT, Thomas HD, Kahn $S$, Kyle $S$, et al. Therapeutic potential of poly(ADP-ribose) polymerase inhibitor AG014699 in human cancers with mutated or methylated BRCA1 or BRCA2. J Natl Cancer Inst 2011;103:334-46.

18. Coleman RL, Oza AM, Lorusso D, Aghajanian C, Oaknin $A$, Dean A, et al. Rucaparib maintenance treatment for recurrent ovarian carcinoma after response to platinum therapy (ARIEL3): a randomised, double-blind, placebocontrolled, phase 3 trial. Lancet 2017;390:1949-61.

19. Eisenhauer EA, Therasse P, Bogaerts J, Schwartz LH, Sargent $D$, Ford $R$, et al. New response evaluation criteria in solid tumours: revised RECIST guideline (version 1.1). Eur J Cancer 2009;45:228-47

20. Rustin GJ, Vergote I, Eisenhauer E, Pujade-Lauraine E, Quinn $M$, Thigpen T, et al. Definitions for response and progression in ovarian cancer clinical trials incorporating RECIST 1.1 and CA 125 agreed by the Gynecological Cancer Intergroup (GCIG). Int J Gynecol Cancer 2011;21:419-23. 